\title{
The impact of a nurse practitioner-led symptom clinic on emergency department use in cancer patients
}

\author{
Shanthi Sivendran, MD, MSCR, ${ }^{a}$ Rachel Holliday, BS, ${ }^{a}$ Ronald Guittar, ${ }^{a}$ Christine Cox, \\ $\mathrm{RN}, \mathrm{BSN},{ }^{\mathrm{a}}$ and Kristina Newport, $\mathrm{MD}^{\mathrm{b}}$
}

${ }^{2}$ Ann B Barshinger Cancer Institute, Penn Medicine at Lancaster General Health, and ${ }^{b}$ Palliative Medicine Consultants, Hospice and Community Care, Lancaster, Pennsylvania

Background Emergency department (ED) use and hospitalization is distressing to cancer patients and drives up the cost of health care. A growing body of evidence demonstrates that more than half of those visits may be avoidable.

Objective To examine the impact of a nurse practitioner (NP)-led, physician-supervised, outpatient symptom management clinic on ED use.

Methods We conducted a retrospective review of ED encounters to quantify the frequency of ED use by oncology patients at a community cancer institute 6 months before (October 2012-March 2013) and after (April-September 2013) the initiation of an NP-staffed symptom management clinic.

Results The highest use of the ED and supportive clinic was among patients with advanced cancer, most commonly with lung or breast cancer, who were receiving cytotoxic chemotherapy. Uncontrolled symptoms of shortness of breath, pain, weakness, fever, nausea, vomiting, and diarrhea commonly led to ED visits. Despite instituting the NP-staffed symptom management clinic to manage those symptoms, there was a $17.9 \%$ increase in ED use. However, of the patients seen by the NP, $95 \%$ may have avoided hospitalization.

Limitations Retrospective study

Conclusions Our study identifies a high-risk population of patients who use the ED frequently. NP-led clinics could aggressively manage the symptom burden of these patients and potentially reduce ED visits as other studies have demonstrated. Although our study did not directly demonstrate this, we have identified weaknesses of care delivery in our clinic that could be optimized. In addition, we have demonstrated that the majority of patients seen for acute symptoms by an NP avoided and ED visit.

$\mathrm{E}$ mergency department (ED) use and hospitalization across the continuum of cancer treatment are common and distressing issues for patients and their families. Use of these services is recognized as an indicator of poor quality end-oflife care, but studies have demonstrated frequent use of them in patients with advanced cancer and particularly in those who are close to the end of life. ${ }^{1-3}$ There is growing evidence that more than $50 \%$ of ED visits are secondary to cancer symptoms and that nearly $23 \%$ of visits could be avoided. ${ }^{4,5}$ Early identification of these patients and aggressive outpatient symptom management may reduce ED use and hospitalization. This improved quality of care could also have a positive impact on the high cost of end-of-life cancer care. Different models of care have been explored to provide outpatient supportive treatment to cancer patients, including nurse practitioner (NP)-driven symptom management clinics.
In this study, we explored the characteristics of high users of emergency department services and the impact of an NP-led, physician-supervised, symptom management clinic on the total frequency of emergency department visits.

\section{Methods}

We conducted a retrospective review of ED encounters to improve quality of care and quantify the frequency of ED use by cancer patients at a community cancer institute. The review period was from October 1, 2012 to September 30, 2013, and a total of 425 patient charts were audited to evaluate ED use by patients who were actively receiving chemotherapy, radiation therapy, or combination therapy. Chart abstractions were completed for each encounter to determine if the patient had received cancer treatment within 30 days of the ED visit. Only patients who were receiving active treatment were included

Accepted for publication December 2, 2015. Correspondence: Shanthi Sivendran MD, MSCR; ssivendran2@lghealth.org. Disclosures: The authors report no disclosures or conflicts of interest. JCSO 2016;14:268-272. (2016 Frontline Medical Communications. doi: 10.12788/jcso.0227. 
in the quality review. Metrics for each patient were collected through review of history, physical, and provider office notes in the electronic medical record. Patient disease site, stage, and treatment modality were collected for each encounter. Additional data about the ED encounter were collected from the emergency department provider notes and progress notes associated with the admission. Data points collected for each ED encounter included: contact with an oncology provider prior to the ED encounter, chief complaint/symptom, admission disposition, and oncology provider visits completed prior to the ED encounter.

From this data we sought to quantify ED use in our patient population both before and after initiation of an NP-led, physician-supervised symptom management clinic. The clinic exists within a large cancer institute affiliated with a community hospital. There are 8 dedicated medical oncologists at the institute. The clinic was staffed by $1 \mathrm{NP}$ with dedicated slots for symptom management 5 days a week during business hours. Physicians saw their own patients urgently when the NP was not present. Referrals to the clinic were made either by the managing physician or based on needs identified during patient calls to the nurse triage service. The ED data were first analyzed to describe the use of ED care by oncology patients receiving active treatments for a 12 -month period. The data on ED use were then divided into 2 groups. Those encounters that occurred in the 6 months immediately before the initiation of the symptom management clinic (October 2012March 2013), were compared with those in 6 months after the launch of the clinic (April 2013-September 2013).

\section{Results}

A total of $425 \mathrm{ED}$ encounters by patients under treatment were identified during a period of 12 months. Of those, 195 encounters occurred in the 6 months before the initiation of the symptom management clinic, and 230 encounters occurred in the 6 months after its initiation. There was a $17.9 \%$ increase in $\mathrm{ED}$ use during the 6 months after initiation of a symptom management clinic (Figure 1).

Before and after initiation of the symptom management clinic, the most common disease site was lung (22.1\% of study population), with a total of $94 \mathrm{ED}$ encounters during the 12 months, followed by breast $(13.2 \%$ of total population), with a total of 56 encounters (Table). Colon cancer and multiple myeloma also ranked in the top 5 sites both before and after symptom management, as well as collectively. Patients with stage IV disease were the highest users of ED services (36.4\% of the total population) both before and after initiation of the symptom management clinic. The majority of patients $(95.8 \%)$ were receiving chemotherapy alone (Table). Of the remaining patients, $2.3 \%$ were receiving a combination of radiation and chemotherapy, and $1.9 \%$ were receiving radiation therapy alone. This did not differ significantly in the before and after symptom management populations.

Pain was the most common complaint among the patients who used the emergency department. A total of 81 patients (19.1\%) went to the ED because of pain, followed by 67 patients (15.8\%) for shortness of breath, 54 (12.7\%) for fever, 54 (12.7\%) for weakness, and 37 (8.7\%) for nausea, vomiting, and diarrhea. Before the symptom management clinic, the top 5 oncology-related symptoms reported in the ED were shortness of breath $(36,18.5 \%)$, pain $(29,14.9 \%)$, fever $(23,11.8 \%)$, weakness $(23,11.8 \%)$, and chest pain $(18,9.2 \%)$. In the 6 months after the clinic was initiated, the symptoms were pain $(52,22.6 \%)$, shortness of breath $(31,13.5 \%)$, fever $(31,13.5 \%)$, weakness (31, $13.5 \%)$, and nausea, vomiting, and diarrhea (21, 9.1\%).

Most patients did not contact a provider (including nurse navigators or nursing triage phone lines) before their ED encounter. In all, only $25.6 \%$ of the total population did so, with $23.1 \%$ of patients having a phone call documented before their ED visit in the 6-month period before the symptom management clinic. There was a slight increase in contact with a provider to $27.8 \%$ in the 6 -month period after the clinic was initiated, but the increase was not statistically significant.

Disposition after the ED visit was reviewed for each encounter. Of the total ED encounters, 232 patients (54.6\%) were admitted to an inpatient unit, 16 (3.8\%) were admitted under observation status, and the remaining 177 patients (41.6\%) were discharged directly from the ED.

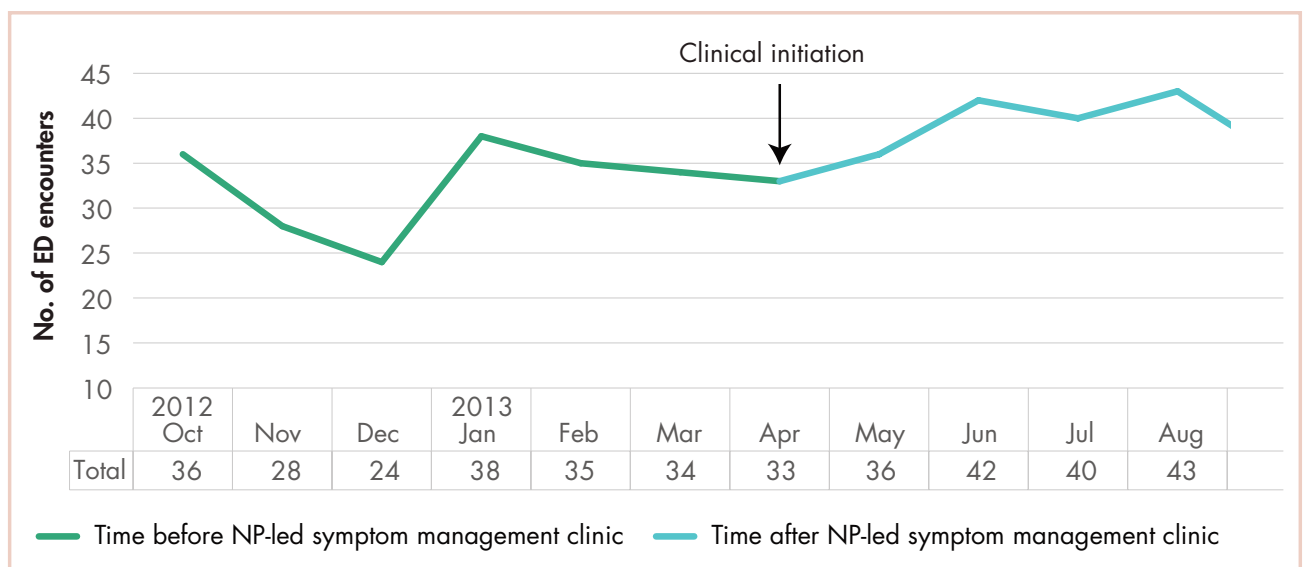

FIGURE 1 Total number of emergency department encounters per month for oncology patients receiving treatment during the 12-month review period. 


\begin{tabular}{|c|c|c|c|c|c|}
\hline \multirow{2}{*}{\multicolumn{2}{|c|}{ Before clinic initiation }} & \multirow{2}{*}{\multicolumn{2}{|c|}{ After clinic initiation }} & \multirow{2}{*}{\multicolumn{2}{|c|}{ Total }} \\
\hline & & & & & \\
\hline Characteristic & n (\%) & Characteristic & n (\%) & Characteristic & $n(\%)$ \\
\hline \multicolumn{6}{|c|}{ Top 5 sites $^{a}$} \\
\hline Lung & $41(21)$ & Lung & $53(23)$ & Lung & $94(22.1)$ \\
\hline Breast & $24(12)$ & Breast & $32(13.9)$ & Breast & $56(13.2)$ \\
\hline Lymphoma & $19(9.7)$ & Colon & $22(9.6)$ & Colon & $34(8)$ \\
\hline Multiple myeloma & $15(7.7)$ & Ovarian & $20(8.7)$ & Lymphoma & $26(6.1)$ \\
\hline Colon & $12(6.2)$ & Multiple myeloma & $11(4.8)$ & Multiple myeloma & $26(6.1)$ \\
\hline \multicolumn{6}{|c|}{ Stage(TNM) } \\
\hline I & $5(2.6)$ & I & $13(5.7)$ & I & $18(4.2)$ \\
\hline$\|$ & $12(6.2)$ & $\|$ & $13(5.7)$ & $\|$ & $25(5.9)$ \\
\hline III & $20(10.3)$ & III & $52(22.6)$ & III & 72 (16.9) \\
\hline IV & $67(34.4)$ & IV & $86(37.4)$ & IV & $153(36)$ \\
\hline UNK & $34(17.4)$ & UNK & $35(15.2)$ & UNK & 69 (16.2) \\
\hline NA & $57(29.2)$ & NA & $31(13.5)$ & NA & $88(20.7)$ \\
\hline \multicolumn{6}{|c|}{ Treatment type } \\
\hline Chemotherapy & $187(95.9)$ & Chemotherapy & $220(95.7)$ & Chemotherapy & 407 (95.8) \\
\hline Radiation & $3(1.5)$ & Radiation & $5(2.2)$ & Radiation & $8(1.9)$ \\
\hline Combination & $5(2.6)$ & Combination & $5(2.2)$ & Combination & $10(2.6)$ \\
\hline \multicolumn{6}{|c|}{ Top 5 symptoms } \\
\hline Shortness of breath & $36(18.5)$ & Pain & $52(22.6)$ & Pain & 81 (19.1) \\
\hline Pain & 29 (14.9) & Fever & $31(13.5)$ & Shortness of breath & $67(15.8)$ \\
\hline Fever & $23(11.8)$ & Shortness of breath & $31(13.5)$ & Fever & $54(12.7)$ \\
\hline Weakness & $23(11.8)$ & Weakness & $31(13.5)$ & Weakness & $54(12.7)$ \\
\hline Chest Pain & $18(9.2)$ & Nausea/vomiting/diarrhea & $21(9.1)$ & Nausea/vomiting/diarrhea & $37(8.7)$ \\
\hline \multicolumn{6}{|c|}{ Contact with oncology before ED encounter } \\
\hline Yes & $45(23.1)$ & Yes & $64(27.8)$ & Yes & $109(25.6)$ \\
\hline No & $143(73.3)$ & No & $156(67.8)$ & No & $299(70.1)$ \\
\hline Other & $7(3.6)$ & Other & $10(4.3)$ & Other & $17(4)$ \\
\hline \multicolumn{6}{|c|}{ Admission disposition } \\
\hline ED-Discharge home & $84(43.1)$ & ED-Discharge home & $93(40.4)$ & ED-Discharge home & $177(41.6)$ \\
\hline ED-Admission & $103(52.8)$ & ED-Admission & $129(56.1)$ & ED-Admission & $232(54.6)$ \\
\hline ED-Observation & $8(4.1)$ & ED-Observation & $8(3.5)$ & ED-Observation & $16(3.8)$ \\
\hline
\end{tabular}

Similar disposition percentages occurred before and after the symptom management clinic (Table).

Time of day and day of week of the ED encounters were determined. Figure 2 displays the distribution of total emergency department encounters across 24 hours, across the 3 nursing shifts, and the number of $\mathrm{ED}$ visits by day of week.
Most ED visits occurred on a Monday (total of 75 patient encounters), with Saturday and Sunday having the second and third highest number of visits (69 for Saturday encounters and 65 for Sunday encounters, respectively). The peak time of day for ED use was $8 \mathrm{pm}$, and when stratified across nursing shifts, most patients (212) were seen during the evening shift $(3 \mathrm{pm}-11 \mathrm{pm})$, with the second-highest 
period of use occurring during the day shift $(7 \mathrm{am}-3 \mathrm{pm})$ and the lowest level of use occurring during the night shift (11 pm-7 am). This finding was consistent for both before and after initiation of the symptom management clinic.

As part of the quality review, each $\mathrm{ED}$ encounter was reviewed to establish if the patient had completed an office visit with an oncology provider, and if so, how close the visit had been to the ED encounter. The majority of patients reviewed had had an office visit with an oncology provider within 30 days of the ED encounter $(84.6 \%$ before clinic initiation and $84.8 \%$ after; Figure 3). However, after initiation of the symptom management clinic, only 20 of the 195 patients seen by an oncology provider before the ED encounter had been seen in the symptom management clinic by the nurse practitioner. Specifically, during the second 6-month review period, 415 patients were seen by the NP for acute symptom management. Of those patients, the aforementioned 20 were seen by the NP before to an $\mathrm{ED}$ visit. That means possibly 395 patients (95\%) who had been seen by the NP had avoided hospitalization.

\section{Discussion}

In 2011, the Institute of Medicine issued a report advising that advanced-practice registered nurses (APRNs) should be partnering with other health care clinicians to practice to the full extent of their training. ${ }^{6}$ APRNs have played an increasingly important role in oncology by both managing patients with cancer and providing palliative care and symptom management. ${ }^{7}$ In the United States, there is 1 oncologist for every 141 newly diagnosed cancer patients, but there is only 1 palliative care physician for every 1,200 patients with a serious illness. ${ }^{8}$ Thus, using APRNs in the outpatient oncology setting for urgent symptom management support may be a way of avoiding ED visits for symptom treatment. Several studies have explored the role of APRN-led supportive care clinics embedded in oncology practices and have demonstrated benefit in providing urgent access for symptom management and in some cases, reducing rates of hospitalization. ${ }^{9-11}$

Our study findings are supported by other findings showing that the highest level of ED and supportive clinic use is in advanced cancer patients, most commonly in those with lung or breast cancer who are receiving cytotoxic che-

motherapy. ${ }^{5,9,12-15}$ In addition, the uncontrolled symptoms of shortness of breath, pain, weakness, fever, nausea, vomiting, and diarrhea which commonly led to ED visits in our patient population were consistently the top sources of distress in other published studies s, $^{5,12-14}$ Of those, pain, nausea, and vomiting have been identified in 2 studies as potentially avoidable reasons for hospitalization. ${ }^{4,5}$ Despite instituting an NP-staffed symptom management clinic to manage the aforementioned symptoms, we retrospectively found a $17.9 \%$ increase in ED use. This suggests that increased access to urgent care visits alone is not enough to prevent avoidable hospitalizations.

Most of the patients in our study who presented to the ED did not contact their oncology provider before or after the institution of the symptom management clinic. Although most of the patients had an office visit with their provider within 30 days before ED presentation, fewer than $10 \%$ of the patients had subsequent visits with the NP. In addition, $41 \%$ of the patients who presented to the ED were treated and discharged from the ED, which suggests that many of these could have been managed 
in an outpatient setting. Finally, of the patients seen by the NP for acute symptoms, 95\% did not have an ED encounter, suggesting that if these clinics were to be used with greater deliberation, it could reduce avoidable hospitalizations. Although the structure of our NP-staffed symptom management clinic did not reduce ED use, other institutions have demonstrated positive results. In one study, a weekly NP-managed symptom management clinic was instituted for patients who were receiving intensive chemoradiotherapy for oropharyngeal cancer. Although that doubled the number of visits to the outpatient clinic, the hospitalization rate was cut by more than half. ${ }^{11}$ This suggests that a more structured visit schedule is more effective than as-needed visits for patients with a high symptom burden. Other studies have emphasized the benefits of the symptom management clinic through patient education before starting chemotherapy and education reinforcing this during chemotherapy. ${ }^{9}$ We have incorporated this strategy in our approach in an effort to optimize the services of our own clinic.

We identified a high-risk population of patients who

\section{References}

1. Earle CC, Park ER, Lai B, Weeks JC, Ayanian JZ, Block S. Identifying potential indicators of the quality of end-of-life cancer care from administrative data. J Clin Oncol. 2003;21:1133-1138.

2. Earle CC, Neville BA, Landrum MB, et al. Evaluating claims-based indicators of the intensity of end-of-life cancer care. Int J Qual Health Care. 2005;17:505-509.

3. Morden NE, Chang CH, Jacobson JO, et al. End-of-life care for Medicare beneficiaries with cancer is highly intensive overall and varies widely. Health Aff (Millwood). 2012;31:786-976.

4. Brooks GA, Abrams TA, Meyerhardt JA, et al. Identification of potentially avoidable hospitalizations in patients with GI cancer. J Clin Oncol. 2014;32:496-503.

5. Delgado-Guay MO, Kim YJ, Shin SH, et al. Avoidable and unavoidable visits to the emergency department among patients with advanced cancer receiving outpatient palliative care. J Pain Symptom Manage. 2015;49:497-504.

6. The Future of Nursing: Leading Change, Advancing Health. Washington (DC):2011.

7. Murphy-Ende K. Advanced practice nursing: reflections on the past, issues for the future. Oncol Nurs Forum. 2002;29:106-112.

8. Morrison RS, Augustin R, Souvanna P, Meier DE. America's care of serious illness: a state-by-state report card on access to palliative care in our nation's hospitals. J Palliat Med. 2011;14:1094-1096. are high users of the ED. NP-staffed symptom management clinics could aggressively manage the symptom burden of these patients and possibly reduce ED visits, as other findings have demonstrated. Although we did not directly demonstrate that, we have shown that the majority of patients seen acutely by an NP potentially avoid hospitalization, and we identified weaknesses of care delivery in our clinic that could be optimized. These include scheduling regular visits with the NP for symptomatic patients until control or resolution of symptoms and educating our patients on the availability of this clinic. In addition, as Monday, Saturday, and Sunday were the peak days for ED use, timing-scheduled NP visits for our at-risk population to occur later in the week may reduce the number of visits to the ED when our cancer institute is closed. Finally, consideration of extended access to our infusion room facilities may reduce the use of the ED during evening hours, another peak use time. Future work should include optimizing the role and practice of NP-driven symptom management clinics to reduce ED use and increase quality of life for cancer patients.

9. Meisenberg BR, Graze L, Brady-Copertino CJ. A supportive care clinic for cancer patients embedded within an oncology practice. J Community Support Oncol. 2014;12:205-208.

10. Ruegg TA. A nurse practitioner-led urgent care center: meeting the needs of the patient with cancer. Clin J Oncol Nurs. 2013;17:E52-57.

11. Mason H, DeRubeis MB, Foster JC, Taylor JM, Worden FP. Outcomes evaluation of a weekly nurse practitioner-managed symptom management clinic for patients with head and neck cancer treated with chemoradiotherapy. Oncol Nurs Forum. 2013;40:581-586

12. Barbera L, Taylor C, Dudgeon D. Why do patients with cancer visit the emergency department near the end of life? CMAJ. 2010;182:563-568.

13. Mayer DK, Travers D, Wyss A, Leak A, Waller A. Why do patients with cancer visit emergency departments? Results of a 2008 population study in North Carolina. J Clin Oncol. 2011;29:2683-2688.

14. McKenzie H, Hayes L, White K, et al. Chemotherapy outpatients' unplanned presentations to hospital: a retrospective study. Support Care Cancer. 2011;19:963-969.

15. Henson L, Gao W, Higginson I, et al. Emergency department attendance by patients with cancer in the last month of life: a systematic review and meta-analysis. Lancet. 2015;385(suppl 1):S41. 